\title{
EHMTI-0177. Evidence for plastic brain morphometric changes during the migraine cycle
}

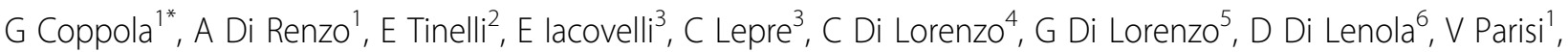 \\ F Pauri $^{3}$, G Fiermonte ${ }^{3}$, F Bianco $^{3}$, F Pierelli ${ }^{6}$ \\ From 4th European Headache and Migraine Trust International Congress: EHMTIC 2014 \\ Copenhagen, Denmark. 18-21 September 2014
}

\section{Background}

Neurophysiological investigations have demonstrated that there are distinctive fluctuations in the brain's electric signals between the ictal and interictal periods of recurrent migraine. Whether structural plasticity of the brain is also an important feature of episodic migraine remains unresolved.

\section{Aim}

We therefore investigated the possibility that there are fluctuations over time in whole brain grey matter morphometry of patients affected by episodic migraine without aura (MO).

\section{Method}

Twenty-four patients with untreated MO underwent MRI scans (3-Tesla Siemens Verio) during $(\mathrm{n}=10)$ or between attacks $(\mathrm{n}=14)$ and were compared to a group of 15 healthy volunteers (HV). We then performed voxelbased-morphometry (VBM) analysis of structural T1-weighted MRI scans to determine if changes in brain structure were observed over the course of the migraine cycle.

\section{Results}

During the interictal phase, MO patients had a significantly lower grey matter (GM) density within the right inferior parietal lobule, right temporal inferior gyrus, right superior temporal gyrus, and left temporal pole than did HV. During attacks, GM density increased within the left temporal pole, bilateral insula, and right lenticular nuclei, but no areas exhibited decreased GM density.

Department of Neurophysiology of Vision and Neurophthalmology, G.B.

Bietti Foundation-IRCCS, Roma, Italy

Full list of author information is available at the end of the article

\section{Conclusion}

The morphometric GM changes between ictal and interictal phases reported in the present study suggest that abnormal structural plasticity may be an important mechanism of migraine pathology. Given the functional neuroanatomy of these areas, our findings suggest that migraine is a condition associated with global dysfunction of multisensory integration and memory processing.

No conflict of interest.

\section{Authors' details}

'Department of Neurophysiology of Vision and Neurophthalmology, G.B. Bietti Foundation-IRCCS, Roma, Italy. ${ }^{2}$ Department of Neurology and Psychiatry Neuroradiology section, Sapienza University of Rome, Roma, Italy. ${ }^{3}$ Department of medico-surgical sciences and biotechnologies Neurology section, Sapienza University of Rome, Roma, Italy. ${ }^{4}$ Department of Neurology, Don Carlo Gnocchi Onlus Foundation, Milano, Italy. ${ }^{5}$ Department of Systems Medicine, University of Rome "Tor Vergata" Laboratory of Psychophysiology Psychiatric Clinic, Roma, Italy. ${ }^{6}$ Department of medico-surgical sciences and biotechnologies, Sapienza University of Rome Polo Pontino, Latina, Italy.

Published: 18 September 2014

doi:10.1186/1129-2377-15-S1-E10

Cite this article as: Coppola et al:: EHMTI-0177. Evidence for plastic brain morphometric changes during the migraine cycle. The Journal of Headache and Pain 2014 15(Suppl 1):E10.

\section{SpringerOpen ${ }^{\odot}$}

(c) 2014 Coppola et al; licensee Springer. This is an Open Access article distributed under the terms of the Creative Commons Attribution License (http://creativecommons.org/licenses/by/2.0), which permits unrestricted use, distribution, and reproduction in any medium, provided the original work is properly cited. 